\title{
MAfint: Modelo Afetivo de Intervenção Tutorial para Ambientes Virtuais de Aprendizagem
}

\author{
Soelaine Rodrigues Ascari ${ }^{1,2}$, Ernani Gottardo ${ }^{3}$, Andrey Ricardo Pimentel ${ }^{1}$ \\ ${ }^{1}$ Programa de Pós-graduação - Universidade Federal do Paraná (UFPR) \\ Curitiba - PR - Brasil \\ ${ }^{2}$ Departamento de Informática - Universidade Tecnológica Federal do Paraná (UTFPR) \\ Pato Branco - PR - Brasil \\ ${ }^{3}$ Instituto Federal de Educação, Ciência e Tecnologia do RS (IFRS) \\ Erechim - RS - Brasil \\ soelainedutfpr.edu.br, ernani.gottardoderechim.ifrs.edu.br, \\ andrey@inf.ufpr.br
}

\begin{abstract}
The teaching and learning process performed virtually has been widespread nowadays with Intelligent Tutoring Systems (ITS), as they can provide immediate and personalized instructions to learners. This personalization can consider the learner affective state to improve teaching strategies. In this work, a tutorial intervention model is presented based on the identification of the type of mathematical error made by the learner. To evaluate the model, an experiment with learners was performed and through the inference of the affective states, the results indicate that personalized interventions provide greater engagement and motivation in comparison to minimal interventions.
\end{abstract}

Resumo. O processo de ensino e aprendizagem realizado de forma virtual tem sido muito difundido atualmente por meio dos Sistemas Tutores Inteligentes (STI), pois podem prover instruções imediatas e personalizadas aos aprendizes. Essa personalização pode considerar o estado afetivo do aprendiz para aperfeiçoar as estratégias de ensino. Neste trabalho é apresentado um modelo de intervenção tutorial com base na identificação do tipo de erro matemático cometido pelo aprendiz. Para avaliar o modelo um experimento com aprendizes foi realizado e por meio da inferência dos estados afetivos os resultados indicam que as intervenções personalizadas favorecem um maior engajamento e motivação em relação às intervenções mínimas.

\section{Introdução}

Em uma de sala de aula presencial geralmente os professores ao constatarem que seus aprendizes apresentam dificuldades, como, por exemplo, na resolução de um exercício, ou mostram-se desmotivados e confusos, realizam adaptações em suas estratégias de ensino com o objetivo de auxiliá-los e mantê-los motivados e engajados. Os STI são softwares educacionais que, por meio da coleta de dados, da observação do comportamento e das ações dos aprendizes realizadas no sistema [dos Santos and Falcão 2017], podem prover auxílio individualizado, instruções imediatas e adaptadas aos aprendizes. Os estados afetivos influenciam diretamente na aprendizagem, motivação e no engajamento do 
aprendiz, positiva ou negativamente [Morais et al. 2017], além de desempenharem papel vital na tomada de decisões, na interação social, percepção, memorização, criatividade e nas atividades de gestão da aprendizagem [Picard 1997]

Outro aspecto que não pode ser negligenciado, e nem visto como algo negativo, é o erro cometido pelo aprendiz no processo de ensino e aprendizagem, seja por falta de conhecimento de conceitos ou mesmo por desatenção [Marczal et al. 2015]. Diagnosticar os erros cometidos pelos aprendizes pode ser considerado como uma oportunidade para construção do conhecimento [Peng and Luo 2009]. Estudos como os de [Movshovitz-Hadar et al. 1987], [Peng and Luo 2009] e [Leite et al. 2012] apresentam modelos ou teorias sobre classificação de erros matemáticos. Com base nos erros, os STI têm a capacidade de identificar de forma individualizada as dificuldades do aprendiz e assim prover uma intervenção tutorial mais apropriada [Leite et al. 2012] e [Marczal et al. 2015].

A intervenção tutorial é importante para manter o controle da situação do tutorial, para resguardar o aprendiz de uma aprendizagem incorreta ou inadequada, além de impedir que o aprendiz explore caminhos (objetivos instrucionais) que não sejam instrucionalmente úteis [Burns and Capps 2013]. O comportamento de um STI é composto por dois laços (loops): um laço externo (outer loop) sobre tarefas e um laço interno (inner loop) sobre etapas. O laço externo é executado uma vez por tarefa, enquanto que o laço interno é executado uma vez para cada etapa [Vanlehn 2006]. O foco desse trabalho é nas intervenções de laço interno.

Neste contexto, este artigo apresenta um modelo de intervenção tutorial que utiliza a classificação de erros matemáticos desenvolvido por [Leite et al. 2012] para identificar os erros cometidos pelos aprendizes e para a indicação das intervenções tutoriais mais adequadas de forma a propiciar um maior engajamento e motivação. Aplica ainda a abordagem do modelo de representação das emoções em quadrantes desenvolvida por [Gottardo and Pimentel 2018] para realizar a inferência dos estados afetivos.

Esse modelo foi implementado em um jogo sobre frações na área da matemática e um experimento foi realizado com dois grupos de aprendizes. A identificação do tipo de erro é realizada por meio do registro do resultado da operação de frações e com base nesses dados o modelo apresenta aos aprendizes de um grupo intervenções especificas, e para o outro grupo intervenções mínimas. A inferência do estado afetivo é realizada pela captura da expressão facial do aprendiz no mesmo momento em que registra o resultado de cada operação. Os resultados do experimento apontam que as intervenções específicas, apresentadas com base no tipo do erro dos aprendizes, propiciam um maior engajamento e motivação em relação às intervenções mínimas.

\section{Trabalhos relacionados}

Com o objetivo de verificar os estudos já realizados no contexto deste trabalho, foi realizado um mapeamento e de todos os trabalhos encontrados, os três que mais contribuíram para o desenvolvimento do presente modelo foram:

O trabalho de [D’Mello et al. 2008], apresenta um agente pedagógico, denominado AutoTutor que sintetiza respostas afetivas por meio de expressões faciais animadas e fala modulada. Quando as emoções tédio, frustração e confusão do aprendiz são inferidas, 
pela expressão facial, o AutoTutor apresenta feedback adaptado. Já [Cooper et al. 2011], apresenta um agente que muda a expressão facial, oferece dicas, vídeos e a opção de alterar o nível de dificuldade do próximo exercício. Isso é realizado a partir da integração de uma variedade de sensores com dados de eventos do aplicativo, de modo que o tutor possa executar intervenções com base no estado afetivo, confiante, interessado, excitado ou frustrado do aprendiz, resolvendo problemas no ambiente de tutoria. Por fim, [Tiam-Lee and Sumi 2018] apresenta um sistema para prática de programação que fornece feedback adaptativo baseado na presença de confusão no aprendiz quando da resolução de exercícios (gerar/alterar complexidade de exercícios e orientações). A inferência do estado afetivo confusão é realizada pela expressão facial. A Tabela 1 apresenta uma compilação das principais características dos trabalhos citados acima.

Tabela 1. Principais características dos trabalhos relacionados.

\begin{tabular}{|l|l|l|l|}
\hline Referência & $\begin{array}{l}\text { Estados afetivos } \\
\text { inferidos }\end{array}$ & Inferência feito por & $\begin{array}{l}\text { Tipos de interven- } \\
\text { ção }\end{array}$ \\
\hline $\begin{array}{l}\text { [DMello et al. } \\
2008]\end{array}$ & $\begin{array}{l}\text { Tédio, Frustração } \\
\text { e Confusão }\end{array}$ & Expressão facial & $\begin{array}{l}\text { Feedback adapta- } \\
\text { tivo }\end{array}$ \\
\hline $\begin{array}{l}\text { [Cooper et al. } \\
2011]\end{array}$ & $\begin{array}{l}\text { Confiante, inte- } \\
\text { ressado, excitado } \\
\text { ou frustrado }\end{array}$ & $\begin{array}{l}\text { Expressão facial, conduti- } \\
\text { vidade da pele, mudanças } \\
\text { de movimento e postura e } \\
\text { pressão no mouse }\end{array}$ & Dicas e mensagens \\
\hline $\begin{array}{l}\text { [Tiam-Lee and } \\
\text { Sumi 2018] }\end{array}$ & Confusão & Expressão facial & $\begin{array}{l}\text { Feedback adapta- } \\
\text { tivo }\end{array}$ \\
\hline
\end{tabular}

Analisando os trabalhos relacionados verificou-se que eles inferem um conjunto pequeno de emoções, geralmente ligados ao estado afetivo de confusão e cada trabalho usa um tipo de intervenção diferente e no trabalho apresentado são usados todos. Sobre as emoções este trabalho contempla a inferência do estado afetivo utilizando a abordagem de representação das emoções em quadrantes [Gottardo and Pimentel 2018], baseado nas emoções básicas do modelo Ekman [Ekman 1992]. Além disso, o modelo agrega uma classificação dos tipos de erros do aprendiz permitindo uma avaliação da correlação entre os tipos de intervenções e mudanças no estado afetivo.

\section{Modelo de Intervenção Tutorial Proposta}

Inicialmente, para o desenvolvimento do modelo de intervenção tutorial proposto pelo presente trabalho, foi necessário realizar um estudo sobre os tipos de intervenções tutoriais aplicados nos ambientes virtuais de aprendizagem. A partir dos trabalhos de [McKendree 1990], [Fleming and Levi 1993], [Hume et al. 1996], [Hannafin et al. 1999], [McLoughlin 2004], [Economides 2005] e [Narciss 2013] foi desenvolvida uma classificação de tipos de intervenções, sendo elas:

- Scaffolding: o termo é utilizado como uma metáfora no processo de aprendizagem, por referir-se ao apoio temporário que é oferecido ao aprendiz para o desenvolvimento de uma atividade. Os subtipos podem ser Conceitual, Processual, Estratégico e Metacognitivo [McLoughlin 2004] e [Hannafin et al. 1999].

- Feedback: são todas as informações pós-resposta que informam os aprendizes sobre seu estado real de aprendizado ou desempenho, além de propiciar ajuda 
ao aprendiz de modo que ele tenha condições de solucionar uma tarefa e com isso progredir no aprendizado. $\mathrm{O}$ feedback pode ser baseado na função ou no conteúdo.

- Baseado na função: Feedback de Confirmação, Corretivo, Explicativo, Diagnóstico e Elaborativo [Fleming and Levi 1993].

- Baseados no conteúdo: Feedback de Conhecimento da resposta, Resposta até que seja correta, Tópico contingente, Resposta contingente, Bug relacionado, Isolamento de atributos [Economides 2005], Conhecimento do desempenho, Conhecimento da resposta correta, Elaborado [Narciss 2013], Violação de condição, Meta ou de objetivo e Combinado [McKendree 1990].

- Dicas: o objetivo é lembrar os aprendizes das informações que se presume serem de seu conhecimento, ou seja, estimular a recordação de fatos que os auxiliem a responder a uma pergunta. As dicas podem ser Informação transmitida (IC - Conveyed Information), Apontar para (PT - Pointing To) e Linha direta de raciocínio (DLR - Directed Line of Reasoning) [Hume et al. 1996].

No jogo de frações matemáticas foram implementadas as intervenções do tipo Dicas, com o subtipo IC, e Feedback, com seus subtipos explicativo, de meta ou de objetivo, corretivo, resposta contingente e conhecimento da resposta correta. A intervenção do tipo Scaffolding não foi aplicada devido ao formato/estilo das operações apresentada no jogo. Para efeito de análise foram considerados os tipos e não os subtipos de intervenções. Essas intervenções foram definidas considerando o resultado de um experimento prévio aplicado a 34 aprendizes de duas escolas municipais do quinto ano do ensino fundamental, com idades entre 10 e 12 anos. Nesse experimento os aprendizes interagiram com o jogo de frações matemáticas resolvendo operações com frações. A partir disso, foram registrados os erros, os estados afetivos e as intervenções tutoriais aplicadas. Após o experimento os dados foram analisados para verificar quais intervenções, com quais tipos de erros mais auxiliaram os aprendizes na resolução das operações.

A Figura 1 é uma representação do modelo de intervenção tutorial que indica intervenções tutoriais a partir da identificação do tipo de erro do aprendiz.

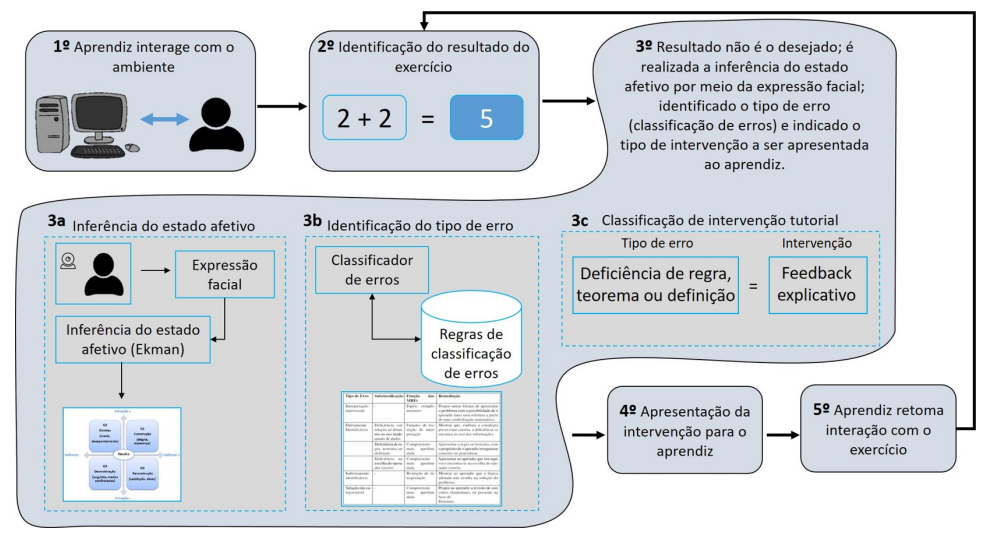

Figura 1. Modelo de inervenção tutorial.

No momento em que o aprendiz responder o exercício $\left(1^{\circ}\right)$ o modelo identifica se a resposta apresentada é a esperada ou não $\left(2^{\circ}\right)$. Se a resposta estiver correta o ambiente 
carrega um novo exercício. Caso contrário, o ambiente realiza uma análise para identificar qual é o tipo de erro cometido pelo aprendiz, e simultaneamente captura a imagem facial do aprendiz. Para a identificação do tipo de erro (3.a) foi utilizada a classificação de erros matemáticos, desenvolvida por [Leite et al. 2012]. Os tipos de erros contidos na classificação são: Interpretação equivocada da linguagem, Diretamente identificáveis, Indiretamente identificáveis e Solução não categorizável.

A inferência do estado afetivo é realizada com base na expressão facial capturada do aprendiz por uma câmera padrão de acordo com as emoções básicas do modelo de Ekman [Ekman 1992]. O modelo de inferência utiliza uma abordagem de representação das emoções em quadrantes, conforme a Figura 2 [Gottardo and Pimentel 2018]. Os quadrantes formados pelas dimensões valência (eixo horizontal) e ativação (eixo vertical) que foram nomeados como Q1, Q2, Q3, Q4, além de um estado Neutro (QN). Esses quadrantes agregam um conjunto de emoções que tem potencial de impactar o processo de aprendizagem.

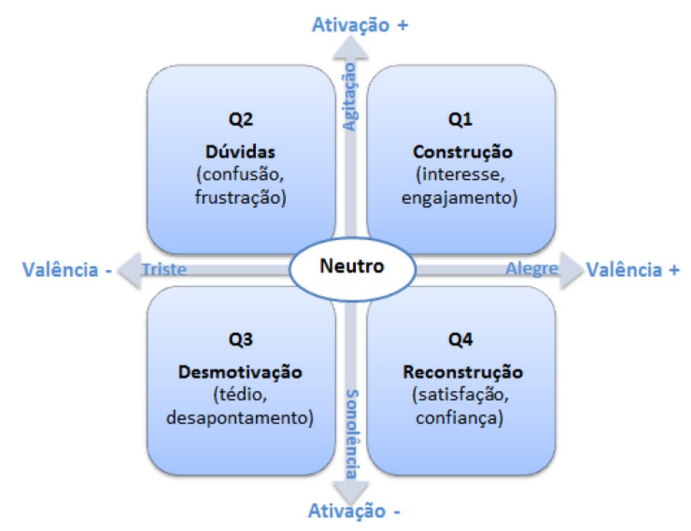

Figura 2. Modelo de representação das emoções em quadrantes.

Fonte: [Gottardo and Pimentel 2018].

Imediatamente após detectar o tipo de erro e inferir o estado afetivo (3c), uma intervenção específica, ou mínima, é exibida para o aprendiz em forma de texto em linguagem natural com o objetivo de auxiliá-lo na resolução do exercício em questão. $\mathrm{O}$ aprendiz após receber a intervenção tutorial, pode resolver o exercício novamente ou encerrar o uso do ambiente. Se obtiver êxito o ambiente carrega um novo exercício, caso contrário retorna à terceira etapa. Dessa forma, o objetivo do modelo é apresentar para os aprendizes intervenções tutoriais específicas que os auxiliem na resolução dos exercícios, além de mantê-los motivados. Para avaliar o modelo um experimento foi realizado e é descrito na próxima seção.

\section{Experimento}

Com a finalidade de verificar a viabilidade do modelo desenvolvido e analisar seus resultados um experimento foi realizado com aprendizes utilizando um jogo de operações sobre frações matemáticas. O jogo desenvolvido para esse experimento consiste na apresentação de 22 operações de frações que considera as quatro operações básicas: soma, subtração, multiplicação e divisão, essas divididas em quatro níveis de dificuldade. Participaram do experimento 40 aprendizes de uma Escola Municipal, de duas turmas do 
IX Congresso Brasileiro de Informática na Educação (CBIE 2020)

Anais do XXXI Simpósio Brasileiro de Informática na Educação (SBIE 2020)

quinto ano do ensino fundamental com idades entre 10 e 12 anos, sendo 18 do sexo feminino e 22 do sexo masculino. O experimento foi realizado entre os meses de novembro e dezembro de 2019.

O experimento foi dividido em três etapas: pré-teste, interação com jogo de frações e pós-teste. Na primeira etapa, pré-teste, os aprendizes foram divididos aleatoriamente em dois grupos: Grupo Experimental (GE) e Grupo de Controle (GC), a partir das notas de uma lista de exercícios impressa sobre frações aplicada e corrigida pela professora da turma. Na sequência foi aplicado o teste de Kolmogorov-Smirnov para verificar a normalidade dos dados (notas), o qual foi confirmada. Foi verificado ainda a homogeneidade dos grupos na média (teste t) e na variância (teste f). Ambos os testes indicaram homogeneidade, ou seja, os dados não evidenciaram diferença significativa, na média e na variância, entre os dois grupos. Com um nível de significância $\alpha=0,05$, teste $\mathrm{t}=0,2550$ e teste $\mathrm{f}=1,0543$ (variância). A partir das listas de exercícios utilizadas no pré-teste foram identificados os erros cometidos pelos aprendizes, e com o auxílio de uma professora da área de matemática foi possível identificar e classificar esses erros de acordo com a classificação de tipos de erros matemáticos de [Leite et al. 2012].

Após a formação do GE e do GC, os aprendizes foram convidados a participar da fase de interação com o jogo de frações. Essa etapa foi realizada no laboratório de informática da escola. Como o jogo apresenta intervenções tutoriais diferentes para cada grupo, optou-se por trabalhar com os aprendizes do GC e do GE separadamente, e enquanto um grupo estava no laboratório de informática, o outro permanecia na sala de aula com a professora desenvolvendo outras atividades. Assim, foram realizadas duas sessões com cada turma com tempo médio de 40 minutos.

Para os aprendizes do GC o jogo apresenta intervenções tutoriais mínimas com duas categorias, correta e incorreta, ou seja, o jogo apresenta apenas se o aprendiz acertou ou errou a operação. Para os aprendizes do GE apresenta intervenções tutoriais específicas, do tipo Dicas ou Feedback, considerando o modelo desenvolvido. Assim, no segundo grupo, quando o aprendiz seleciona uma resposta errada é verificado o tipo de erro, feita a inferência do estado afetivo por meio da expressão facial e na sequência é realizada a intervenção tutorial. Após o aprendiz do GE selecionar uma resposta errada pela quarta vez consecutiva, em uma determinada questão, é apresentada uma intervenção específica com a resposta correta e qual o erro cometido. Para os aprendizes do GC é apresentada apenas a intervenção mínima. Nos dois grupos as intervenções tutoriais foram apresentadas por um avatar. Cabe ressaltar que todos os aprendizes concluíram o experimento e que os grupos receberam as mesmas operações durante a interação com o jogo.

$\mathrm{Na}$ última etapa, pós-teste, os aprendizes responderam uma nova lista de exercícios sobre frações e assim como no pré-teste a professora aplicou, corrigiu e atribuiu nota. Importante destacar que o objetivo do pós-teste foi de comparar os resultados do pré-teste com os do pós-teste para verificar se as intervenções tutoriais específicas do GE os ajudaram a obter um melhor resultado em relação aos aprendizes do GC.

Duas hipóteses foram formuladas para o experimento. A primeira hipótese verifica se a média das notas do pós-teste dos aprendizes do GE foi significativamente, a um nível $\alpha=0,05$, maior que a média das notas do pós-teste do GC. A segunda hipótese formulada verifica se a aplicação de intervenções específicas associadas ao contexto deste trabalho 
IX Congresso Brasileiro de Informática na Educação (CBIE 2020)

Anais do XXXI Simpósio Brasileiro de Informática na Educação (SBIE 2020)

propicia engajamento/motivação maior se comparado com as intervenções mínimas.

\section{Resultados do Experimento e Discussões}

Para a primeira hipótese, média das notas do pós-teste, foi aplicado um teste t para grupos independentes, os resultados sinalizaram positivamente, com um grau de confiança de $95 \%$, com $\mathrm{p}$-value $=0,0348$ e teste $\mathrm{t}=1,8674$, ou seja, os dados revelaram diferença significativa $(\alpha=0,05)$ em termos da média das notas entre o GC e o GE. Assim, como $\mathrm{p}<\alpha$, há evidências para dizer que essa diferença se deve as intervenções tutoriais específicas apresentas para o GE que auxiliaram os aprendizes a obterem um melhor resultado na aprendizagem.

Para responder a segunda hipótese algumas informações serão apresentadas sobre o experimento que foi descrito na seção anterior. Foram registradas 1361 ocorrências de estados afetivos durante o jogo. Dessas, 1244 (92,1\%) são ocorrências identificadas, ou seja, que foi possível inferir a emoção do aprendiz por meio da expressão facial e mapear no quadrante correspondente, de acordo com a abordagem de representação de emoções por quadrante. Não foram identificadas $117(8,6 \%)$ ocorrências. Os principais motivos da não identificação da face foram a posição do aprendiz em relação a câmera, a mão na frente da boca ou do rosto. A média de ocorrências de estados afetivos por aprendiz foi de 31,1 com desvio padrão de 6,4. A Tabela 2 apresenta o número e o valor \% de ocorrências por quadrante em cada um dos grupos, com exceção do quadrante Q3.

Tabela 2. Total de ocorrências por quadrante.

\begin{tabular}{|l|l|l|l|l|}
\hline Quadrantes & GC & GE & \% GC & \% GE \\
\hline Q1 & 16 & 59 & $2,5 \%$ & $\mathbf{9 , 7 \%}$ \\
\hline Q2 & 10 & 17 & $1,6 \%$ & $2,8 \%$ \\
\hline Q3 & 0 & 0 & $0,0 \%$ & $0,0 \%$ \\
\hline Q4 & 10 & 16 & $1,6 \%$ & $2,6 \%$ \\
\hline QN & 602 & 514 & $94,4 \%$ & $84,8 \%$ \\
\hline Total & 638 & 606 & $100 \%$ & $100 \%$ \\
\hline Média & $127,6(\sigma=265,3)$ & $121,2(\sigma=220,7)$ & & \\
\hline
\end{tabular}

Pode ser observado que o quadrante com o maior número de ocorrências de estados afetivos foi o QN, nos dois grupos, com $94,4 \%$ para o GC e $84,8 \%$ para o GE. O predomínio do estado afetivo neutro era esperado para essa atividade e alinha-se com o resultado de outros trabalhos nessa área [Gottardo and Pimentel 2018]. Na sequência o quadrante Q1 apresenta 2,5\% de ocorrências para o GC e 9,7\% para o GE. Isso significa que os aprendizes do GE obtiveram mais ocorrências do quadrante Q1 em relação ao GC. Esse quadrante identifica a ocorrência de estados afetivos como alegria, engajamento e motivação, estados esses desejados para melhorar a experiência e os resultados de aprendizagem.

A Tabela 3 apresenta a quantidade de ocorrências de intervenções para cada grupo. Essas intervenções foram apresentadas para os aprendizes dos GC e GE sempre que a resposta selecionada para uma operação estava incorreta.

O GE obteve um valor percentual de $35,5 \%$ de acertos com o uso das intervenções específicas em relação ao total de ocorrências do grupo, e 64,5\% de erros, enquanto o GC 
Tabela 3. Total de intervenções.

\begin{tabular}{|l|l|l|l|l|l|}
\hline & $\begin{array}{l}\text { Qtde de ocor- } \\
\text { rências de } \\
\text { intervenções }\end{array}$ & $\begin{array}{l}\text { Qtde de } \\
\text { acertos }\end{array}$ & $\begin{array}{l}\text { Qtde de er- } \\
\text { ros }\end{array}$ & $\begin{array}{l}\text { \% de acer- } \\
\text { tos por } \\
\text { grupo }\end{array}$ & $\begin{array}{l}\text { \% de erros } \\
\text { por grupo }\end{array}$ \\
\hline GC & 264 & 64 & 200 & $24,2 \%$ & $\mathbf{7 5 , 8 \%}$ \\
\hline GE & 217 & $\mathbf{7 7}$ & 140 & $\mathbf{3 5 , 5 \%}$ & $64,5 \%$ \\
\hline Total & 481 & 141 & 340 & & \\
\hline Média & $241(\sigma=33,2)$ & $71(\sigma=9,2)$ & $170(\sigma=42,4)$ & & \\
\hline
\end{tabular}

obteve $24,2 \%$ de acertos com o uso de intervenções mínimas e 75,8\% de erros em relação ao total de ocorrências. Observa-se que os aprendizes do GE tiveram mais acertos que os aprendizes do GC. Isso indica que as intervenções recebidas pelos aprendizes do GE ajudaram na resolução das operações de frações do jogo em relação ao outro grupo que não recebeu as mesmas intervenções.

A Tabela 4 apresenta as mudanças de quadrante por tipo de intervenção em cada grupo.

Tabela 4. Mudança de quadrante x intervenção.

\begin{tabular}{|c|c|c|c|c|}
\hline & \multicolumn{2}{|l|}{ GC } & \multicolumn{2}{|l|}{ GE } \\
\hline & $\begin{array}{l}\text { Intervenção } \\
\text { mínima }\end{array}$ & $\begin{array}{l}\text { \% sobre o } \\
\text { total de mu- } \\
\text { danças }\end{array}$ & $\begin{array}{l}\text { Intervenção } \\
\text { específica }\end{array}$ & $\begin{array}{l}\text { \% sobre } \\
\text { o total de } \\
\text { mudanças }\end{array}$ \\
\hline Mudou de estado para Q1 & 5 & $11,4 \%$ & 18 & $36,7 \%$ \\
\hline Mudou de estado para Q2 & 3 & $6,8 \%$ & 5 & $10,2 \%$ \\
\hline Mudou de estado para Q3 & 0 & $0,0 \%$ & 0 & $0,0 \%$ \\
\hline Mudou de estado para Q4 & 6 & $13,6 \%$ & 4 & $8,2 \%$ \\
\hline Mudou de estado para QN & 30 & $68,2 \%$ & 22 & $44,9 \%$ \\
\hline Total & 44 & $100,0 \%$ & 49 & $100,0 \%$ \\
\hline
\end{tabular}

A maior mudança foi dos quadrantes Q1, Q2 ou Q4 para o quadrante QN. É possível observar ainda que as intervenções específicas do GE obtiveram um percentual maior $(36,7 \%)$ em relação à intervenção mínima do GC $(11,4 \%)$. Essa informação é uma indicação que intervenções específicas, como do tipo Dicas e Feedback, dadas ao GE nesse contexto ajudaram os aprendizes a manterem-se mais motivados e engajados que os aprendizes do GC que receberam intervenções mínimas.

As intervenções específicas do tipo Dicas e Feedback do GE geraram 18 mudanças de algum quadrante para Q1, além de manter 12 vezes o quadrante Q1 após a intervenção. Dessas houve 6 ocorrências de mudanças de quadrantes diferentes para Q1 a partir de intervenções do tipo "Feedback"e 9 ocorrências de intervenções onde o aprendiz se manteve em Q1. Ainda no GE ocorreram 12 ocorrências de mudanças de quadrantes diferentes para Q1 a partir de intervenções do tipo "Dicas"e 3 ocorrências de intervenções em que o aprendiz se manteve em Q1. Já para o GC houve somente 5 mudanças de algum quadrante para o Q1 a partir de uma intervenção mínima (certo/errado) e não foi registrada ocorrência em que o quadrante Q1 se manteve. A partir desse resultado é possível concluir que a segunda hipótese foi atendida. 
IX Congresso Brasileiro de Informática na Educação (CBIE 2020)

Anais do XXXI Simpósio Brasileiro de Informática na Educação (SBIE 2020)

\section{Conclusão}

Neste trabalho foi apresentado um experimento com aprendizes do quinto ano do ensino fundamental aplicando um jogo de frações matemáticas, em que a partir da identificação do tipo do erro da operação de frações matemáticas é apresentado ao aprendiz uma intervenção tutorial imediata do tipo específica: Dicas ou Feedback. Além disso foi inferido o estado afetivo em cada resposta do aprendiz para verificar se essas intervenções contribuem para um maior engajamento em relação a uma intervenção mínima.

Os resultados iniciais apresentados neste trabalho mostram-se promissores, mesmo considerando a limitação no número de aprendizes envolvidos na pesquisa. Estes resultados poderiam ser utilizados para implementação de funcionalidades específicas em ambientes virtuais de aprendizagem para melhorar a experiência de aprendizagem, aumentar o engajamento e motivação, buscando impactar positivamente o desempenho dos aprendizes.

Nesse contexto, os resultados indicam que as intervenções específicas contribuíram para uma maior ocorrência de mudança e permanência dos aprendizes de outros quadrantes para o Q1. O quadrante Q1, valência e ativação positiva, indica o estado afetivo das emoções positivas do aprendiz, como, alegria, motivação e engajamento, emoções que favorecem e que têm potencial de impactar o processo de aprendizagem. Os resultados indicam ainda uma melhora no aprendizado que pode ser decorrente de um estado motivacional melhor.

Observando que as intervenções específicas ajudaram a melhorar os resultados dos aprendizes, uma indicação para trabalhos futuros é agregar ao modelo apresentado que o estado afetivo inferido pela representação de quadrantes fosse considerada para a indicação da intervenção tutorial, ou seja, a intervenção seria apresentada com base no tipo de erro e do estado afetivo do aprendiz.

\section{Referências}

Burns, H. L. e Capps, C. G. (2013). Foundations of intelligent tutoring systems: An introduction. Foundations of intelligent tutoring systems, page 1.

Cooper, D. G., Arroyo, I., e Woolf, B. P. (2011). Actionable affective processing for automatic tutor interventions. In New Perspectives on Affect and Learning Technologies, pages 127-140. Springer New York.

dos Santos, D. C. V.-B. e Falcão, T. P. (2017). Acompanhamento de alunos em ambientes virtuais de aprendizagem baseado em sistemas tutores inteligentes. In (Simpósio Brasileiro de Informática na Educação-SBIE), volume 28, page 1267.

D’Mello, S., Jackson, T., Craig, S., Morgan, B., Chip-Man, P., White, H., Person, N., Kort, B., Kaliouby, R. E., Picard, R., e Graesser, A. (2008). Autotutor detects and responds to learners affective and cognitive states. Workshop on Emotional and Cognitive Issues at the Int. Conf. Intelligent Tutoring Systems. Montreal, Canada, pages 306-308.

Economides, A. A. (2005). Adaptive feedback evaluation. In Proceedings of the 5th WSEAS International Conference on Distance Learning and Web Engineering. 
IX Congresso Brasileiro de Informática na Educação (CBIE 2020)

Anais do XXXI Simpósio Brasileiro de Informática na Educação (SBIE 2020)

Ekman, P. (1992). An argument for basic emotions. Cognition and Emotion, 6(3-4):169200.

Fleming, M. L. e Levi, W. H. (1993). Instructional message design: Principles from the behavioral and cognitive sciences. Educational technology.

Gottardo, E. e Pimentel, A. (2018). Reconhecimento e adaptação à dinâmica de estados afetivos relacionados à aprendizagem. In Anais do XXIX Simpósio Brasileiro de Informática na Educação (SBIE 2018).

Hannafin, M., Land, S., e Oliver, K. (1999). Open learning environments: Foundations, methods, and models. Instructional-design theories and models: A new paradigm of instructional theory, 2:115-140.

Hume, G., Michael, J., Rovick, A., e Evens, M. (1996). Hinting as a tactic in one-on-one tutoring. Journal of the Learning Sciences, 5(1):23-47.

Leite, M. D., Pimentel, A. R., e Pietruchinski, M. H. (2012). Remediação de erros baseada em múltiplas representações externas e classificação de erros aplicada a objetos de aprendizagem inteligentes. In Simpósio Brasileiro de Informática na Educação-SBIE, volume 23.

Marczal, D., Direne, A., Pimentel, A., e Krynski, E. M. (2015). FARMA: Uma ferramenta de autoria para objetos de aprendizagem de conceitos matemáticos. In Anais dos Workshops do IV Congresso Brasileiro de Informática na Educação (CBIE 2015).

McKendree, J. (1990). Effective feedback content for tutoring complex skills. Human-Computer Interaction, 5(4):381-413.

McLoughlin, C. (2004). Achieving excellence in teaching through scaffolding learner competence. Seeking Educational Excellence, pages 9-10.

Morais, F., Silva, J. D., Reis, H., Isotani, S., e Jaques, P. (2017). Computação afetiva aplicada à educação: uma revisão sistemática das pesquisas publicadas no brasil. In Anais do XXVIII Simpósio Brasileiro de Informática na Educação (SBIE 2017).

Movshovitz-Hadar, N., Zaslavsky, O., e Inbar, S. (1987). An empirical classification model for errors in high school mathematics. Journal for research in mathematics Education, pages 3-14.

Narciss, S. (2013). Designing and evaluating tutoring feedback strategies for digital learning environments on the basis of the interactive tutoring feedback model. Digital Education Review, 23:7-26.

Peng, A. e Luo, Z. (2009). A framework for examining mathematics teacher knowledge as used in error analysis. For the learning of mathematics, 29(3):22-25.

Picard, R. (1997). Affective computing. Cambridge: MIT Press.

Tiam-Lee, T. J. e Sumi, K. (2018). Adaptive feedback based on student emotion in a system for programming practice. In Intelligent Tutoring Systems, pages 243-255. Springer International Publishing.

Vanlehn, K. (2006). The behavior of tutoring systems. International journal of artificial intelligence in education, 16(3):227-265. 